\title{
Unraveling Entanglement
}

\author{
André R.R. Carvalho ${ }^{1,2}$, Marc Busse ${ }^{1}$, Olivier Brodier ${ }^{1}$, Carlos Viviescas ${ }^{1}$, and Andreas Buchleitner ${ }^{1}$ \\ ${ }^{1}$ Max-Planck-Institut für Physik komplexer Systeme, \\ Nöthnitzer Strasse 38, 01187 Dresden, Germany and \\ ${ }^{2}$ Dept. of Physics, Faculty of Science, Australian National University, ACT 0200, Australia
}

(Dated: April 2, 2006)

\begin{abstract}
We show that the time evolution of entanglement under incoherent environment coupling can be faithfully recovered by monitoring the system according to a suitable measurement scheme.
\end{abstract}

PACS numbers: 03.67.-a,03.67.Mn,03.65.Yz,42.50.Lc

Quantum information processing requires the ability to produce entangled states and coherently perform operations on them. Under realistic laboratory conditions, however, entanglement is degraded through uncontrolled coupling to the environment. It is of crucial practical importance to quantify this degradation process [1-3], though also extremely difficult in general, due to the intricate mathematical notions upon which our understanding of entanglement relies [4-6]. Up to now, no general observable is known which would complement such essentially formal concepts with a specific experimental measurement setup.

In the present Letter, we come up with a dynamical characterization of entanglement, through the continuous observation of a quantum system which evolves under incoherent coupling to an environment. We show that, at least for small, yet experimentally relevant systems, there is an optimal measurement strategy to monitor the entanglement of the time evolved, mixed system state. Mixed state entanglement is then given as the average entanglement of the pure states generated by single realisations of the optimal measurement-induced, stochastic time evolution.

Consider a bipartite quantum system composed of subsystems $A$ and $B$, interacting with its environment. Due to this coupling, an initially pure state $\left|\Psi_{0}\right\rangle$ of the composite system will evolve into a mixed state $\rho(t)$, in a way governed by the master equation

$$
\dot{\rho}=-\frac{i}{\hbar}[H, \rho]+\sum_{k=1}^{N} \mathcal{L}_{k} \rho,
$$

where the Hamiltonian $H$ generates the unitary system dynamics. The superoperators $\mathcal{L}_{k}$ describe the effects of the environment on the system, and, for a Markovian bath, have the standard form [7]

$$
\mathcal{L}_{k} \rho=\frac{\Gamma_{k}}{2}\left(2 J_{k} \rho J_{k}^{\dagger}-J_{k}^{\dagger} J_{k} \rho-\rho J_{k}^{\dagger} J_{k}\right),
$$

where the operators $J_{k}$ depend on the specific physical situation under study.

To extract the time evolution of entanglement under this incoherent dynamics, one solution is to evaluate a given entanglement measure $M(\rho)$ for the solution $\rho(t)$, at all times $t$. One starts from one of the known pure state measures $M(\Psi)[5,6,8]$, together with a pure state decomposition of $\rho$,

$$
\rho=\sum_{i} p_{i}\left|\Psi_{i}\right\rangle\left\langle\Psi_{i}\right|
$$

where the $p_{i}$ are the positive, normalized weights of each pure state $\left|\Psi_{i}\right\rangle$. The most naive generalization for a mixed state would then be to consider the average

$$
\bar{M}=\sum_{i} p_{i} M\left(\Psi_{i}\right)
$$

which, however, is not suitable, since the decomposition (3) is not unique: $\bar{M}$ would thus give rise to different values of entanglement for different valid decompositions of $\rho$ [9], inconsistently with the general requirements for a bona fide entanglement measure $[5,6]$. The proper definition of $M(\rho)$ therefore is the infimum of all possible averages $\bar{M}[10]$, but holds two main drawbacks: (i) it turns into a hard numerical problem for higher dimensional or multipartite systems, and, (ii) even for bipartite qubits, where analytical solutions for some measures $M(\rho)$ are known [8], there is no obvious interpretation of this optimal decomposition, in physical terms.

Our approach here will be to avoid the direct use of the mixed state solutions $\rho(t)$, by substituting them by physically motivated ensembles of pure states. To do this, instead of solving the evolution equation for the density operator, we will follow a stochastic time evolution $[9,11-13]$ of the initially pure state. This combines randomly occurring quantum jumps, defined by the action of the operators $J_{k}$ in Eq. (2), with periods of continuous evolution, generated by a non-hermitian effective Hamiltonian $H_{\text {eff }}=H-i \hbar \sum_{k} \Gamma_{k} J_{k}^{\dagger} J_{k} / 2$. The resulting quantum trajectories, see Fig. 1, are known to provide the same result as the master equation, on averaging over many independent realisations. Moreover, a single trajectory can be understood as the information accessible through the continuous experimental monitoring of the system [12-15]. In other words, one possible decomposition of the form (3) is given by the set of the possible outcomes $\left|\Psi_{i}\right\rangle$ of different runs of one and the same experiment, after a monitoring time $t$.

Note, however, that the stochastic time evolution induced by the jump operators $J_{k}$ does not necessarily yield the optimal decomposition which minimises $\bar{M}$. 


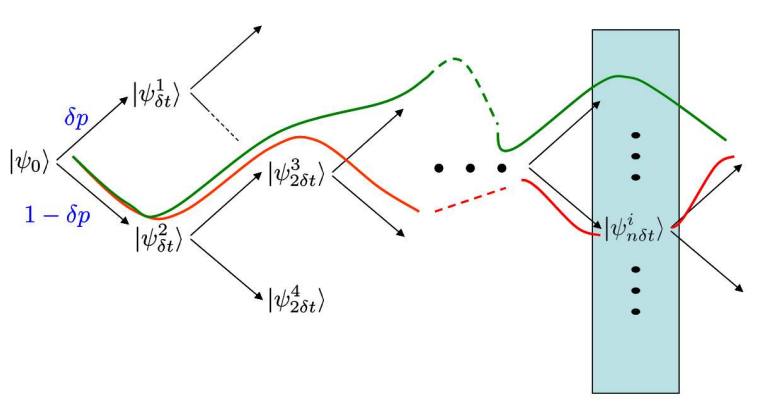

FIG. 1: Scheme of a stochastic time evolution generated by the action of a jump operator $J$ : At each time step $\delta t, \delta p$ gives the probability for a jump into the state $\left|\Psi_{\delta t}^{1}\right\rangle$, while $(1-\delta p)$ indicates the probability for a continuous evolution of the system in the state $\left|\Psi_{\delta t}^{2}\right\rangle$. Iterative application of this stochastic jump process generates different stochastic pure state trajectories, which define a pure state decomposition of the time evolved density matrix $\rho(t)$, at $t=n \delta t$.

Though, the choice of the jump operators is not unique, and new jumps defined as $L_{k, \pm}=\left(\mu_{k} \pm \tilde{J}_{k}\right) / \sqrt{2}$, with $\tilde{J}_{k}=\sum_{i} U_{k i} J_{i}$, (left) unitary $U$, and complex $\mu_{k}$, leave Eq. (2) unaltered. Each such choice of jump operators produces a different unraveling of the same master equation (2), i.e., a different decomposition of $\rho(t)$. In order to find the infimum of (4), we have to search for that type of monitoring which reproduces the desired optimal decomposition. It is by no means obvious that such monitoring exists, since the set of physically realizable decompositions of $\rho$ is only a subset of all possible decompositions [16]. However, in the sequel of this paper, we present strong evidence for experimentally relevant scenarios, suggesting that such a measurement prescription indeed can always be found, for a given initial state, and given environment coupling.

We focus on the dynamics of bipartite two-levels systems, where our results can readily be compared with known analytical solutions $[6,8,17]$. We assume that each subsystem interacts independently with its own environment, and consider those situations where decoherence is induced by dissipation (zero temperature reservoir) or by dephasing. For simplicity, we set all decay rates $\Gamma_{k} \equiv \Gamma$ in Eq. (2). We start with the general jump operators $L_{k, \pm}$ as defined above, and search for the ones which yield the optimal solution. The first step in such procedure is to follow all possible states $\left|\Psi_{\delta t}\right\rangle$ of the system, at a short time $\delta t$ after the initial time $t_{0}=0$, and to calculate the average measure $\bar{M}(\delta t)$. Since the possible states $\left|\Psi_{\delta t}^{k}\right\rangle, k=1 \ldots N$, which can be reached after this short time interval are given by the action of the $N$ jump operators, plus the state $\left|\Psi_{\delta t}^{N+1}\right\rangle$ which results from continuous evolution (see Fig. 1), the expression for the average measure reads

$$
\bar{M}(\delta t)=\left(1-\sum_{k=1}^{N} \delta p_{k}\right) M\left(\Psi_{\delta t}^{N+1}\right)+\sum_{k=1}^{N} \delta p_{k} M\left(\Psi_{\delta t}^{k}\right),
$$

with $p_{k}=\delta t\left\langle\Psi_{0}\left|L_{k \pm}^{\dagger} L_{k \pm}\right| \Psi_{0}\right\rangle$ the probability of detecting a jump induced by $L_{k \pm}$. Eq. (5) can be minimised over the different possible unravelings, what ensures that the short time behaviour of the average measure is optimal.

To illustrate this in more detail, let us first consider the case of spontaneous emission, which corresponds to the fundamental limiting factor for the coherent evolution of atomic qubits. The Lindbladians in Eq. (2) are given by $J_{1}=\sqrt{\Gamma}\left(\sigma_{-}^{(1)} \otimes \mathbb{1}\right)$, and $J_{2}=\sqrt{\Gamma}\left(\mathbb{1} \otimes \sigma_{-}^{(2)}\right)$, where $\sigma_{-}^{(i)}$ is the deexcitation operator of the $i$ th qubit. General jump operators can be parametrised as

$$
\left(\begin{array}{c}
L_{1, \pm} \\
L_{2, \pm}
\end{array}\right)=\frac{1}{\sqrt{2}}\left(\begin{array}{c}
\mu_{1} \\
\mu_{2}
\end{array}\right) \pm \frac{1}{\sqrt{2}} U\left(\begin{array}{c}
J_{1} \\
J_{2}
\end{array}\right)
$$

with the unitary $2 \times 2$ matrix [22]

$$
U=e^{i \chi}\left(\begin{array}{cc}
\alpha e^{i \theta} & \beta e^{i \varphi} \\
-\beta e^{-i \varphi} & \alpha e^{-i \theta}
\end{array}\right) .
$$

Our entanglement measure will be the concurrence $c(\Psi)=\left|\left\langle\Psi^{*}\left|\sigma_{y} \otimes \sigma_{y}\right| \Psi\right\rangle\right|[8]$ of bipartite, pure qubit states (analogous results are obtained with other measures such as the entanglement of formation $[5,8])$.

For a general initial state $\left|\Psi_{0}\right\rangle=\psi_{00}|00\rangle+\psi_{01}|01\rangle+$ $\psi_{10}|10\rangle+\psi_{11}|11\rangle, c\left(\Psi_{0}\right)=2\left|\psi_{01} \psi_{10}-\psi_{00} \psi_{11}\right|$. Using these definitions in Eq. (5), together with the explicit form of the operators $L_{k, \pm}$, Eq. (6), one gets

$$
\begin{aligned}
\bar{c}(\delta t) & =\left[1-\delta t\left(2 \mu^{2}+\Gamma\right)\right] c\left(\Psi_{0}\right) \\
& +2 \delta t\left|\mu^{2} e^{2 i \chi} r\left(\Psi_{0}\right)+\Gamma \alpha \beta e^{i(\theta+\varphi)} \psi_{11}^{2}\right| \\
& +2 \delta t\left|\mu^{2} e^{2 i \chi} r\left(\Psi_{0}\right)-\Gamma \alpha \beta e^{-i(\theta+\varphi)} \psi_{11}^{2}\right|,
\end{aligned}
$$

where $r\left(\Psi_{0}\right)=\psi_{00} \psi_{11}-\psi_{01} \psi_{10}$, and we set $\mu_{1}=\mu_{2}=$ $\mu e^{i \chi}$. The optimal unraveling has to minimize $\bar{c}$ over the free parameters of the transformations $(6,7)$.

Let us first consider initial states with $\psi_{11}=0$. Equation (8) then reduces to

$$
\bar{c}(\delta t)=(1-\Gamma \delta t) c\left(\Psi_{0}\right),
$$

which is independent of the unraveling. For such states, the ensemble of quantum trajectories always remains within the subspace spanned by $\{|00\rangle,|01\rangle,|10\rangle\}$. Hence, Eq. (9) holds for all times and coincides with the known analytical solution for $c(\rho(\delta t))$ [6]. Entanglement decay of $\psi_{00}|00\rangle+\psi_{01}|01\rangle+\psi_{10}|10\rangle$ due to spontaneous emission can thus be detected by any measurement strategy, and, in particular, simply by the continuous monitoring of the spontaneously emitted photons.

For initial states including the $|11\rangle$ component, one has to deal with (8) in its full glory, and the optimal parameter values are found to be $\alpha=\beta=1 / \sqrt{2}, \theta+\varphi=\pi / 2=$ 


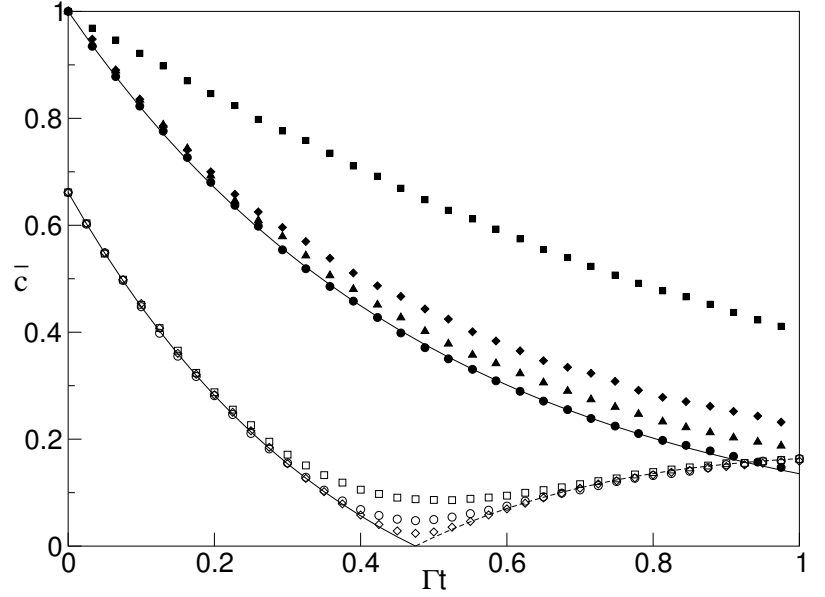

FIG. 2: Time evolution of the bipartite mixed state concurrence for initial states $\left|\Psi_{0}\right\rangle=(|00\rangle+|11\rangle) / \sqrt{2}(\bar{c}(t=0)=1)$ and $\left|\Psi_{0}\right\rangle=\sqrt{1 / 8}|00\rangle+\sqrt{7 / 8}|11\rangle(\bar{c}(t=0) \simeq 0.7)$, under incoherent coupling to a zero temperature environment. Continuous lines represent exact solutions; filled squares stem from a randomly chosen unraveling. Symbols show the results for improving unravelings with increasing $\left|\mu_{1}\right|=\left|\mu_{2}\right|=0.8$ (filled diamonds), 1.0 (filled pyramids), 3.0 (filled circles), 4.0 (open squares), 7.0 (open circles), and 15.0 (open diamonds). The dashed line shows the time evolution of $|\Lambda(t)|=\left|\lambda_{1}-\sum_{i=2}^{4} \lambda_{i}\right|$ beyond the disentanglement time $t_{d} .1000$ realizations were used to generate the unraveling data, in all cases.

$-2 \chi+\sigma, \sigma=\arg \left(\psi_{11}^{2} / r\left(\Psi_{0}\right)\right), \mu \geq \sqrt{\left|\psi_{11}^{2} / r\left(\Psi_{0}\right)\right| / 2}$, provided $\left|\Psi_{0}\right\rangle$ is nonseparable (for arbitrary separable initial states, $\alpha=0$ or $\beta=0$ enforce $\bar{c}(\delta t)=0$ ). While such choice guarantees the correct short-time behavior, it is not always sufficient for the correct long-time evolution. Further optimizations, now in a parameter space reduced by the above constraints, are needed. Specifically in the present case of spontaneous emission, increasing the value of $\mu$ rapidly improves the agreement with the exact result, over longer and longer time scales. On the time interval monitored in Fig. 2, the exact solution is faithfully recovered for $\mu \geq 3$. For general initial states, the convergence in $\mu$ can be understood and estimated by optimizing the increments of $\bar{c}$ at subsequent time steps $m \delta t, m$ integer. In contrast, a non-optimal choice of $\mu$ and $U$ produces a completely misleading result.

The entanglement of initial states with $0<c\left(\Psi_{0}\right)<$ $2\left|\psi_{11}\right|^{2}$ vanishes at finite times $t_{d}$. Since $\bar{c}\left(t_{d}\right)$ is obtained as a weighted sum of non-negative pure state concurrences, each single term has to vanish identically for the optimal unraveling. This is, the optimal unraveling must generate an ensemble of separable pure states at the disentanglement time $t_{d}$. In practice, this is achieved in the limit $\mu \rightarrow \infty$ and $\delta t \rightarrow 0$, as illustrated in Fig. 2 for optimal $U$ and increasing $\mu$ [23]. Once complete separability is reached, entanglement cannot revive under purely incoherent dynamics, and the optimal unraveling for times $t>t_{d}$ has to be replaced by the trivial unraveling for separable initial states (see above), to reflect the true en-

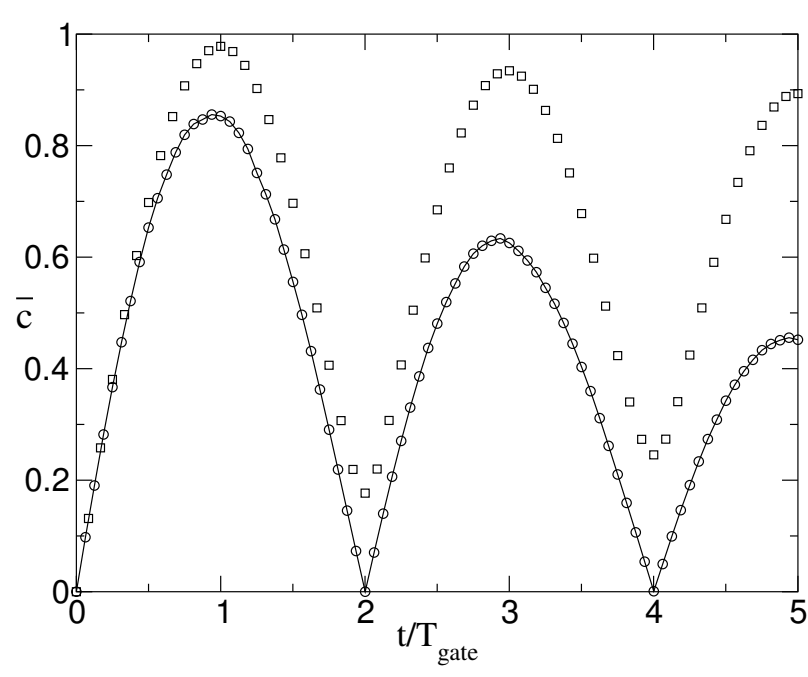

FIG. 3: Concurrence of two qubits initially prepared in $\left|\Psi_{0}\right\rangle=(|00\rangle+|10\rangle) / \sqrt{2}$, under the action of the CNOT gate Hamiltonian (see text) with dephasing. Open circles show the evolution for an optimal unraveling, in perfect agreement with the exact solution (continuous line), and in pronounced contrast to the result obtained from the least favourable unraveling (open squares).

tanglement evolution. However, and remarkably so, the optimal unraveling defined through the above optimization faithfully follows the time evolution of the modulus of $\Lambda(t)=\lambda_{1}-\sum_{i=2}^{4} \lambda_{i}[24]$, where the $\lambda_{i}$ are the singular values of the matrix $\left\langle\Psi_{k}^{*}\left|\sigma_{y} \otimes \sigma_{y}\right| \Psi_{j}\right\rangle$, constructed from the state decomposition at any time $t$, in particular also for $t>t_{d}$. Note that non-negative values of $\Lambda(t)$ precisely coincide with the explicit expression [8] for the mixed state concurrence of qubit pairs, for $t<t_{d}$, and that vanishing or negative values of $\Lambda(t)$ indicate the state's separability.

More general entanglement dynamics, e.g., with a nontrivial unitary evolution in the presence of incoherent environment coupling, can be unraveled with the above strategy. As an example illustrated in Fig. 3, consider the action of a CNOT gate, generated by the Hamiltonian $H=2 \pi \mathbb{I}_{4}+\frac{\pi}{2} \sigma_{+} \sigma_{-} \otimes\left(\sigma_{x}-\mathbb{I}_{2}\right)$, in contact with a dephasing reservoir. Starting from the separable state $\left|\Psi_{0}\right\rangle=$ $(|00\rangle+|10\rangle) / \sqrt{2}$, the noise-free Hamiltonian generates the maximally entangled state $|\Psi\rangle=(|00\rangle+|11\rangle) / \sqrt{2}$, after a time $T_{\text {gate }}$ However, in the presence of dephasing, the entanglement at $T_{\text {gate }}$ (which we chose to be five times shorter than the decoherence time $1 / \Gamma$ ) will not be maximal, since the target state preparation will not be perfect. Analogously to the above, by optimizing the increments of $\bar{c}$ at $2 \delta t(\bar{c}$ is independent of the unraveling at $\delta t)$, one finds an unraveling which generates an optimal pure state decomposition, in excellent agreement with the exact entanglement evolution obtained from the solution $\rho(t)$. If, instead, one maximizes $\bar{c}$ by suitable choice of the jump operators [18], the resulting unraveling suggests rather distinct entanglement dynamics, with apparently almost 


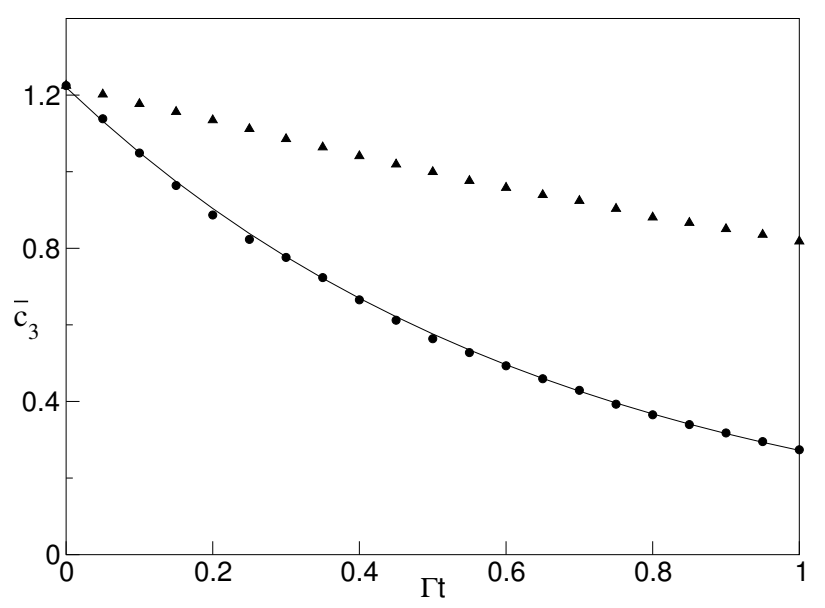

FIG. 4: Concurrence of a tripartite GHZ state under a dephasing environment. Filled circles represent the tripartite concurrence $c_{3}(t)$ obtained upon averaging over 1000 realisations of the optimal unraveling defined by the jump operators $J_{k}=\sqrt{\Gamma} \sigma_{+}^{(k)} \sigma_{-}^{(k)}(k=1,2,3)$, in perfect agreement with the exact solution (solid line). A non-optimal unraveling (pyramids) is shown for comparison.

perfect gate performance at $t=T_{\text {gate }}-$ in pronounced contrast to the correct result.

As a final example, let us consider a simple case of multipartite entanglement - tripartite GHZ or W states, in contact with dephasing or zero temperature environments, respectively. In this case, we choose the generalized concurrence $c_{3}$, which averages over all nontrivial partitions of the given state $[3,6]$, as specific entanglement measure $M . c_{3}$ can be evaluated explicitely $[3,6]$ for tripartite GHZ and W states, under the chosen environment coupling. Once again, we optimize the jump operators $L_{k, \pm}$ at $\delta t$, and obtain excellent agreement with the exact result. Fig. 4 compares the optimal unraveling with a suboptimal one, for a GHZ initial state subject to dephasing. In this exemplary case, the optimal detection is provided by the original jump operators $J_{k}=\sqrt{\Gamma} \sigma_{+}^{(k)} \sigma_{-}^{(k)}, k=1,2,3$. The same applies for $\mathrm{W}$ states.

To conclude, we have shown that mixed state entanglement can be unraveled by a suitable measurement prescription. The latter can be derived from the initial state of the composite quantum system under study, together with the specific type of environment coupling. There is no ambiguity in the definition of mixed state entanglement with respect to different unravelings: the minimal nonclassical correlations needed to characterize a dynamically evolving quantum state are filtered out by an optimal (in general not unique) monitoring prescription, and any in this sense non-optimal unraveling provides a nonoptimal estimate thereof.

Surprisingly, in all examples considered, we so far always succeeded to come up with a time-independent optimal unraveling, i.e., the monitoring strategy does not need to be readapted during the incoherent time evolution. This suggests a kind of uniform convergence of the stochastic average towards the exact solution, and implies a subtle relation between the temporal evolution of entanglement and the ensemble of pure states which can be reached during the single realisations generated by the optimal jump operators.

Future work will have to formulate the general ramifications of the approach outlined here, possibly in the framework of quantum state diffusion theory [19]. In view of possible experimental realizations, finite detection efficiencies as well as general noisy environments [20] need to be addressed.

We thank Florian Mintert, Lajos Diósi, Howard M. Wiseman, Thomas Wellens, Daniel Estève, Armin Uhlmann, and Robin Hudson for constructive suggestions, entertaining discussions and critical remarks.
[1] T. Yu and J. H. Eberly, Phys. Rev. B 66, 193306 (2002).

[2] C. F. Roos, G. P. T. Lancaster, M. Riebe, H. Häffner, W. Hänsel, S. Gulde, C. Becher, J. Eschner, F. SchmidtKaler, and R. Blatt, Phys. Rev. Lett. 92, 220402 (2004).

[3] A. R. R. Carvalho, F. Mintert, and A. Buchleitner, Phys. Rev. Lett. 93, 230501 (2004).

[4] R. F. Werner, Phys. Rev. A 40, 4277 (1989).

[5] D. Bruss, J. Math. Phys. 43, 4237 (2002).

[6] F. Mintert, A. R. R. Carvalho, M. Kuś, and A. Buchleitner, Phys. Rep. 415, 207 (2005).

[7] G. Lindblad, Math. Phys. 48, 119 (1976).

[8] W. K. Wootters, Phys. Rev. Lett. 80, 2245 (1998).

[9] H. Nha and H. J. Carmichael, Phys. Rev. Lett. 93, 120408 (2004).

[10] A. Uhlmann, Phys. Rev. A 62, 032307 (2000).

[11] H. Carmichael, An open systems approach to quantum optics, Lecture Notes in Physics (Springer-Verlag, Berlin, 1993).
[12] K. Mølmer, Y. Castin, and J. Dalibard, J. Opt. Soc. Am. B 10, 524 (1993).

[13] K. Mølmer and Y. Castin, Quant. Semiclass. Opt. 8, 49 (1996).

[14] T. Sauter, W. Neuhauser, R. Blatt, and P. Toschek, Phys. Rev. Lett. 57, 1696 (1986).

[15] H. M. Wiseman and G. J. Milburn, Phys. Rev. A 47, 1652 (1993).

[16] H. M. Wiseman and J. A. Vaccaro, Phys. Rev. Lett. 87, 240402 (2001).

[17] A. R. R. Carvalho, F. Mintert, S. Palzer, and A. Buchleitner (2005), quant-ph/0508114.

[18] M. Gu, S. Parkins, and H. Carmichael, quant$\mathrm{ph} / 0602083$ (2006).

[19] L. Diósi, Phys. Lett. 114A, 451 (1986).

[20] M. Busse, Master's thesis, Ludwig-MaximiliansUniversität München (2006).

[21] T. Wellens and M. Kuś, Phys. Rev. A 64, 052302 (2001). 
[22] A larger number of jump operators can be generated by a rectangular matrix $U$. However, the presently employed square matrix is sufficient for our purposes.

[23] Note that this limit is strictly realized by diffusive quan- tum stochastic differential equations [19].

[24] Which is also given by the smallest eigenvalue of a matrix constructed from the partial transpose of $\rho(t)$ [21]. 